\title{
Doenças sistêmicas e interdisciplinaridade: a importância do diálogo entre o cirurgião dentista e o médico no tratamento e prognóstico de pessoas com enfermidades sistêmicas
}

Nas últimas décadas, tem ficado mais evidente a associação entre doenças sistêmicas e alterações orais. Portanto, é imprescindível que haja comunicação constante entre profissionais médicos e odontólogos, visando um atendimento integral e humanizado e, assim, melhor prognóstico e qualidade de vida do paciente. Com isso, o objetivo deste trabalho é elucidar a importância da abordagem interprofissional entre esses profissionais, com enfoque no tratamento de pessoas com histórico de doenças sistêmicas, como Hipertensão Arterial Sistêmica (HAS), Diabetes Mellitus (DM) e cardiopatias. Sendo assim, foi realizada uma revisão da literatura, com artigos em português e espanhol, entre os anos de 2016 e 2020 . Constatou-se que, na DM, é possível observar associação com alterações na cavidade oral como doença periodontal (DP), alteração do paladar, candidíase oral, xerostomia e halitose. Adicionalmente, constatou-se forte evidência tanto entre a DP e DM quanto com a HAS/cardiopatias apresentando uma interação benéfica a ambas as patologias. Enquanto, por um lado, a hiperglicemia pode predispor o surgimento e agravamento da DP, esta, por outro, possui influência na piora do controle glicêmico. O processo inflamatório sistêmico promovido pela DP mostrou-se associado ao desenvolvimento ou agravamento de doenças cardiovasculares e pacientes que não respondem ao tratamento periodontal, possuem alto risco de doença cardiovascular.

Palavras-chave: Odontólogo; Médicos; Assistência Integral à Saúde; Equipe de Assistência ao Paciente; Doenças Periodontais.

\section{Systemic diseases and interdisciplinary: the importance of dialogue between dental surgeon and doctor in the treatment and prognosis of people with systemic diseases}

In the last decades, the association between systemic diseases and oral disorders has become more evident. Therefore, it is essential that there is constant communication between medical professionals and dentists, aiming at comprehensive and humanized care and, thus, better prognosis and quality of life for the patient. Thus, the objective of this work is to elucidate the importance of the interprofessional approach among these professionals, focusing on the treatment of people with a history of systemic diseases, such as Systemic Arterial Hypertension (SAH), Diabetes Mellitus (DM) and heart diseases. Therefore, a literature review was carried out, with articles in Portuguese and Spanish, between the years 2016 and 2020 . It was found that, in DM, it is possible to observe an association with changes in the oral cavity such as periodontal disease (PD), taste, oral candidiasis, dry mouth and halitosis. In addition, there was strong evidence both between PD and DM and with SAH / heart disease showing a beneficial interaction to both pathologies. While, on the one hand, hyperglycemia can predispose to the onset and worsening of PD, this, on the other hand, has an influence on worsening glycemic control. The systemic inflammatory process promoted by PD has been shown to be associated with the development or worsening of cardiovascular diseases and patients who do not respond to periodontal treatment have a high risk of cardiovascular disease.

Keywords: Dentists; Physicians; Comprehensive Health Care; Patient Care Team; Periodontal Diseases.

Topic: Patologia Clinica e Laboratorial

Received:30/03/2021

Reviewed anonymously in the process of blind peer.

Iasmyn Paranhos de Oliveira (iD)

Universidade de Federal de Juiz de Fora, Brasil

http://lattes.cnpq.br/6106943363148147

http://orcid.org/0000-0003-0750-0326

ia086302@gmail.com

Marcos Lorran Paranhos Leão (iD

Universidade de Pernambuco, Brasil

http://lattes.cnpq.br/7449167140800038

http://orcid.org/0000-0002-6259-2430

upeleao@gmail.com

Carlos Eduardo Pinto de Alcântara (iD

Universidade de Federal de Juiz de Fora, Brasil

http://lattes.cnpq.br/1381994884634996

http://orcid.org/0000-0001-9756-5750

cepalcantara@gmail.com

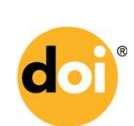

DOI: 10.6008/CBPC2236-9600.2021.002.0014
Approved:10/04/2021
Referencing this:

OLIVEIRA, I. P.; LEÃO, M. L. P.; ALCÂNTARA, C. E. P.. Doenças sistêmicas e interdisciplinaridade: a importância do diálogo entre o cirurgião dentista e o médico no tratamento e prognóstico de pessoas com enfermidades sistêmicas. Scire Salutis, v.11, n.2, p.127-132, 2021. DOI: http://doi.org/10.6008/CBPC2236-9600.2021.002.001 


\section{INTRODUÇÃO}

O Diabetes Mellitus (DM), doença crônica e metabólica, é decorrente de duas possíveis situações, sendo elas: diminuição da produção de insulina pelo pâncreas ou dificuldade do corpo em utilizar, de forma adequada, a insulina que é produzida. Além disso, ela pode ser classificada em dois tipos: o Tipo I tem origem genética, se desenvolvendo ainda na infância, já o Tipo II, tem relação direta com o hábito de vida do indivíduo, como, por exemplo, dieta inapropriada e falta de exercícios físicos, se desenvolvendo, principalmente, na fase adulta. Essa condição aumenta a taxa de açúcar no sangue, levando a um quadro de hiperglicemia, que contribui para a diminuição do fluxo salivar, e consequentemente, proliferação de bactérias acidófilas, como Streptococcus mutans, causador da doença cárie (YAMA et al., 2013; HADLAQ et al., 2017; LATTI et al., 2018).

Além disso, a Hipertensão Arterial Sistêmica (HAS) ocorre quando há aumento da pressão sanguínea nas artérias. Devido a isso, o coração necessita de mais força para conseguir distribuir o sangue para todo o corpo de forma efetiva. Sabe-se que essa condição possui um alto índice de mortalidade se não houver diagnóstico e tratamento eficaz no estágio inicial; ainda, como consequência dos casos de acometimentos assintomáticos, o número de complicações, como acidente vascular cerebral, infarto, aneurisma arterial e insuficiência renal e cardíaca, estão mais frequentes (VERÍSSIMO, 2017; SOUSA et al., 2019; VAISHNAVI et al., 2019). A interação interprofissional entre médicos e dentistas é incomum nessa situação; contudo, estudos atuais mostram evidências sobre a relação entre manifestações orais, como líquen plano e língua geográfica, em pacientes adultos portadores de hipertensão. Além dessas, o tratamento farmacológico pode ocasionar alterações orais, como disgeusia, síndrome da ardência bucal e xerostomia (VERÍSSIMO, 2017; VAISHNAVI et al., 2019).

Com base nos trabalhos disponíveis, observa-se grande associação entre doença periodontal (DP) e alterações sistêmicas. Uma delas é a DM, que é um fator de risco para a periodontite e altera de forma significativa o controle glicêmico do paciente (GRAZIANI et al., 2018). Além disso, há evidências de que a doença periodontal tenha relação com a doença cardiovascular, hipertensão arterial e doenças autoimunes, como lúpus eritematoso sistêmico (MARTIN-CABEZAS et al., 2016; BERLIN-BRONER et al., 2017; RUTTER-LOCHER et al., 2017; WINNING et al., 2017). Ainda, pacientes que não respondem ao tratamento periodontal, possuem alto risco de doença cardiovascular (HOLMLUND et al., 2017). De acordo com as evidências apresentadas, é inquestionável que a saúde geral e a saúde oral são essenciais uma para outra (TSE, 2018; JAVAID et al., 2019; KASSIM et al., 2019; RENNER, 2020).

Em relação ao tratamento dessas doenças crônicas, muitas vezes, são empregados métodos farmacológicos. Por um longo período, constatou-se que alguns medicamentos, usados para tratamento dessas doenças, causam alterações no periodonto, favorecendo a progressão da doença periodontal, além de poder causar crescimento e sangramento gengival e inflamação (HUGHES et al., 2000; PIRES et al., 2017). Ademais, é importante ressaltar, que a interação medicamentosa existente entre o tratamento médico e odontológico, deve ser constantemente observada, bem como, manifestações orais de doenças 
sistêmicas, uma vez, que estas podem ser a primeira ou mais grave característica da patologia (PORTER et al., 2017).

Portanto, devido a inegável relação entre a saúde bucal e a saúde geral, bem como a aparente importância da frequente comunicação entre o médico e o cirurgião dentista, é imprescindível que essa relação seja melhor verificada no âmbito de doenças sistêmicas já conhecidas e amplamente estudadas. Dessa maneira, o objetivo dessa pesquisa é elucidar a importância da abordagem interprofissional entre os profissionais médicos e odontólogos, com enfoque no tratamento de pacientes portadores de doenças sistêmicas, como HAS, DM e cardiopatias.

\section{MEDOTOLOGIA}

Trata-se de um estudo de abordagem qualitativa, de natureza básica, de objetivo exploratório e de procedimento baseado em uma pesquisa bibliográfica, de caráter integrativo $(n=21)$. A busca foi feita utilizando os Descritores em Ciências da Saúde (DeCS): Physicians, Dentists, Hypertension, Diabetes Mellitus e Heart Diseases, sendo filtrada por: artigos publicados entre 2016 e 2020, e nas línguas portuguesa e espanhola. A pesquisa foi feita nas bases de dados SpringerLink, Google Acadêmico, Pubmed e Scielo. De modo que esta é uma pesquisa que utiliza exclusivamente textos científicos já publicados para revisão da literatura científica, não precisa de prévia autorização em nenhum comitê de ética, respaldada pelo parágrafo único do Art. $1^{\circ}$ da resolução 510/2016, resolvida pela Comissão Nacional de Ética em Pesquisa (CONEP), do Conselho Nacional de Saúde (CNS) (CNS e MES, 2016).

\section{RESULTADOS}

Após a análise dos trabalhos levantados, verifica-se a importância do tratamento farmacológico para diversas patologias sistêmicas. Porém, alguns desses medicamentos, podem ocasionar diversas alterações na cavidade bucal. Ainda, alguns estudos mostraram evidências entre a relação das principais classes de fármacos e as manifestações orais, bem como suas interações no tratamento médico e odontológico (tabela 1) (PIRES et al., 2017; PANDOIN et al., 2018).

Tabela 1: Relação entre as classes farmacológicas e manifestações orais, juntamente com suas interações medicamentosas.

\begin{tabular}{|l|l|l|l|}
\hline \multicolumn{2}{|c|}{ Classe farmacológica } & \multicolumn{1}{c|}{ Manifestações orais } & \multicolumn{2}{c|}{ Interação medicamentosa } \\
\hline Anticonvulsivante & $\begin{array}{l}\text { Lesões liquenoides, Granuloma } \\
\text { piogênico extragengival e Hiperplasia } \\
\text { gengival. }\end{array}$ & Opioides & $\begin{array}{l}\text { Aumento da analgesia e depressão } \\
\text { do SNC }\end{array}$ \\
\hline Antidepressivo tricíclico & Diminuição do fluxo salivar & Opioides & $\begin{array}{l}\text { Aumenta a toxicidade e } \\
\text { biodisponibilidade da morfina }\end{array}$ \\
\hline Imunossupressores & $\begin{array}{l}\text { Hiperplasia gengival; } \\
\text { Hiperpigmentação da língua }\end{array}$ & Dipirona & Reduz a ação do medicamento \\
\hline Antimicrobianos & $\begin{array}{l}\text { Placas, Lesões liquenoides e Úlceras } \\
\text { irregulares }\end{array}$ & $\begin{array}{l}\text { Cetorolaco de } \\
\text { Trometamina }\end{array}$ & $\begin{array}{l}\text { Úlceras gastrointestinais ou } \\
\text { convulsões }\end{array}$ \\
\hline Anti-hipertensivo & Hiperplasia gengival & $\begin{array}{l}\text { Diminui o efeito do anti-hipertensivo } \\
\text { dos bloqueadores dos canais de } \\
\text { cálcio }\end{array}$ \\
\hline
\end{tabular}


Doenças sistêmicas e interdisciplinaridade: a importância do diálogo entre o cirurgião dentista e o médico no tratamento e prognóstico de pessoas

OLIVEIRA, I. P.; LEÃO, M. L. P.; ALCÂNTARA, C. E. P.

\begin{tabular}{|l|l|l|l|}
\hline AINEs & Ulceração & $\begin{array}{l}\text { Amoxicilina, } \\
\text { Ampicilina e } \\
\text { Cefalosporina }\end{array}$ & $\begin{array}{l}\text { Nefrotoxicidade, hipersensibilidade, } \\
\text { anafilaxia e alterações } \\
\text { gastrointestinais }\end{array}$ \\
\hline Antipsicótico & $\begin{array}{l}\text { Diminuição do fluxo salivar; } \\
\text { Xerostomia. }\end{array}$ & $\begin{array}{l}\text { Clorpromazina } \\
\text { constipação, enjoos, sonolência e } \\
\text { congestão nasal. }\end{array}$ \\
\hline
\end{tabular}

Fonte: (OLIVEIRA et al., 2020); (PIRES et al., 2017); (PANDOIN et al., 2018).

Outrossim, constata-se que, na DM, as principais alterações que ocorrem na cavidade oral são: doença periodontal, cárie dentária, alteração do sabor, candidíase oral, xerostomia, hipossalivação, lesão periapical e halitose. Em relação à doença periodontal, observa-se que existe uma interação bidirecional com a DM, em que a hiperglicemia e resposta imune, anormal, do hospedeiro, mediante as infecções bucais, predispõem o surgimento e agravamento da DP. Porém, a DP, quando grave, possui grande influência na piora do controle glicêmico do paciente durante anos. Além disso, estudos mostram que a Cardiopatia Isquêmica é a principal causa de morte em DM tipo II, sendo que, quando há comprometimento ventricular esquerdo, devido a esta condição, o DM se torna um importante fator para mortalidade cardiovascular (FENG et al., 2017).

Em relação à HAS, estudos mostram que esta condição por si só e seu tratamento com drogas simpaticomiméticas, são capazes de diminuir o pH salivar, trazendo alteração no paladar, dificuldade na deglutição, predisposição ao desenvolvimento da cárie dental, bem como, alteração da lubrificação e proteção das mucosas do trato gastrointestinal. Em contrapartida, o processo inflamatório sistêmico promovido por condições bucais, como gengivite, periodontite e perda dentária, têm sido associadas ao desenvolvimento ou agravamento do quadro hipertensivo (MUSSANE et al., 2018).

A primeira causa de morte em todo mundo, são as doenças cardiovasculares, e trabalhos têm demonstraram sua significativa relação com a doença periodontal. Apesar de necessitar de estudos mais detalhados, duas hipóteses são apresentadas: a primeira defende a ação direta dos microrganismos, se baseando no fato de espécies bacterianas comumente encontradas nas bolsas periodontais, também terem sido encontradas nas paredes internas dos vasos sanguíneos, no caso de periodontite não tratada. A segunda hipótese se firma na concentração aumentada de granulócitos circulantes no sangue, demonstrando que além da periodontite exacerbada a pessoa possui níveis superiores de inflamação sistêmica, quando comparadas a indivíduos sem DP (RIBEIRO et al., 2016; MANSO et al., 2019).

\section{DISCUSSÃO TEÓRICA}

Os achados deste trabalho corroboram com a literatura já existente na medida em que concorda que alterações sistêmicas como a diabetes mellitus e doenças cardiológicas podem ter apresentações orais e/ou que o tratamento dessas doenças influenciam na saúde bucal e, assim, uma conversa entre os profissionais de ambas as áreas é necessária. Além disso, o tratamento empregado para essas doenças, bem como o seu prognóstico pode ser melhor manejado quando as alterações orais também são levadas em conta (HUGHES et al., 2000; MARTIN-CABEZAS et al., 2016; BERLIN-BRONER et al., 2017; HOLMLUND et al., 2017; PIRES et al., 2017; PORTER et al., 2017; RUTTER-LOCHER et al., 2017; VERÍSSIMO, 2017; WINNING 
et al., 2017; GRAZIANI et al., 2018; VAISHNAVI et al., 2019).

É necessário destacar que esta pesquisa se limitou a analisar os textos científicos já publicados e encontrados nos repositórios nas buscas feitas pelos autores. Assim, este se caracteriza como uma pesquisa secundária. Ainda, teve um enfoque restrito a alguns quadros sistêmicos e, por isso, apresenta limitações quanto aos demais. Contudo, já se percebe a importância da interprofissionalidade do tratamento de todos os pacientes.

Desse modo, novos estudos, que incluam as interações entre os acometimentos sistêmicos e bucais são necessários. Além disso, trabalhos que correlacionam o tratamento e o prognóstico dessas associações devem ser feitos, visando identificar como todas as terapias influenciam e se apresentam nos acometidos, tendo como meta uma visão integral e multifacetada dos pacientes.

\section{CONCLUSÃO}

Portanto, após a análise dos resultados preliminares, consideramos que as manifestações orais das doenças sistêmicas, bem como dos tratamentos para essas enfermidades, devem ser levadas em conta com vista a abarcar a atenção total ao paciente. Tanto os casos em que doenças sistêmicas levem a mudanças orais, quanto em casos que os medicamentos e tratamentos dessas enfermidades alterem a saúde bucal, devem ser completamente analisados para um melhor prognóstico, tratamento e evolução dos pacientes. Ainda, a abordagem interprofissional não é só importante pelo fato de que algumas doenças bucais e sistêmicas compartilham de mesmos fatores de risco, mas, também, por haver relação direta em algumas manifestações clínicas, piora de indicadores de saúde e interação entre os diferentes tipos de tratamentos bucais e sistêmicos.

Assim, consideramos a importância do trabalho em conjunto entre o cirurgião dentista e o profissional médico, no acompanhamento de pessoas com histórico de doenças sistêmicas. Por fim, é indicado um tratamento em que o paciente seja analisado como um indivíduo completo, construindo boa comunicação entre os profissionais, para que, dessa maneira, seja estabelecido um melhor prognóstico e promoção da saúde.

\section{REFERÊNCIAS}

BERLIN-BRONER, Y.; FEBBRAIO, M.; LEVIN, L.. Association between apical periodontitis and cardiovascular diseases: a systematic review of the literature. Int Endod J, v.9, n.50 p.847-859, 2017. DOI: http://doi.org/10.1111/iej.12710

CNS. Resolução № 510, de 07 de abril de 2016. Brasília: 2016.

FENG, A.; PAÑA, Y.; LI, W.. La cardiopatía isquémica en pacientes diabéticos y no diabéticos. Rev Haban Cienc Méd, v.2, n.16, p.217-228, 2017.

GRAZIANI, F.; GENNAI, S.; SOLINI, A.; PETRINI, M.. A systematic review and meta-analysis of epidemiologic observational evidence on the effect of periodontitis on diabetes An update of the EFP-AAP review. J Clin
Periodontol, v.2, n.45, p.167-187, 2018. DOI: http://doi.org/10.1111/jcpe.12837

HADLAQ, E. M.; FARAJ, Z. T.; AL GAMDI, F. M.; AL OBSTHSNI, F. A.; ABUABAT, M. F.; AWAN, K. H.. Early Screening of Diabetes and Hypertension in Primary Care Dental Clinics at King Saud University in Riyadh, Kingdom of Saudi Arabia. J Contemp Dent Pract, v.8, n.18, p.652-659, 2017. DOI: http://doi.org/10.5005/jp-journals-10024-2101

HOLMLUND, A.; LAMPA, E.; LIND, L.. Poor Response to Periodontal Treatment May Predict Future Cardiovascular Disease. J Dent Res, v.7, n.96, p.768-773, 2017. DOI: http://doi.org/10.1177/0022034517701901

HUGHES, F. J.; BARTOLD, P. M.. Periodontal complications of 
prescription and recreational drugs. Periodontol 2000, v.1, n.78, p.47-58, 2018. DOI: http://doi.org/10.1111/prd.12230

JAVAID, Z.; IMTIAZ, U.; KHALID, I.; SAEED, H.; KHAN, R. Q.; ISLAM, M.; SALEEM, Z.; SOHAIL, M. F.; DANISH, Z.; BATOOL, F.; ANWER, N.. A randomized control trial of primary carebased management of type 2 diabetes by a pharmacist in Pakistan. BMC Health Serv Res, v.1, n.19, p.409, 2019. DOI: http://doi.org/10.1186/s12913-019-4274-z

KASSIM, S.; OTHMAN, B.; ALQAHTANI, S.; KAWTHAR, A. M.; MCPHERSON, S. M.; GREENBERG, B. L.. Dentists' attitudes towards chairside medical conditions screening in a dental setting in Saudi Arabia: an exploratory cross-sectional Study. BMC Oral Health, v.1, n.19, p.179, 2019. DOI: http://doi.org/10.1186/s12903-019-0870-x

LATTI, B. R.; KALBURGE, J. V.; BIRAJDAR, S. B.; LATTI, R. G.. Evaluation of relationship between dental caries, diabetes mellitus and oral microbiota in diabetics. J Oral Maxillofac Pathol, v.2, n.22, p.282, 2018. DOI: http://doi.org/10.4103/jomfp.JOMFP 16316

MANSO, I. S.; ANGST, P. D. M.. Quais são as evidências sobre a inter-relação entre a doença periodontal e a hipertensão arterial? Braz J Periodontol, v.2, n.29, p.43-50, 2019.

MARTIN-CABEZAS, R.; SEELAM, N.; PETIT, C.; AGOSSA, K.; GAERTNER, S.; TENENBAUM, H.; DAVIDEAU, J.; HUCK, O. Association between periodontitis and arterial hypertension: a systematic review and meta-analysis. Am Heart J, n.180, p. 98-112, 2016. DOI: http://doi.org/10.1016/i.ahj.2016.07.018

MUSSANE, R. D.; BENEDITO, F. C. D. S.; JOAQUIM, D. C.; RODRIGUES, J. C.; LEITE, A. K. R. D. M.; LEITE, A. C. R. D. M.. Pacientes hipertensos: dos cuidados em saúde ao conhecimento das patologias orais e sua relação com a hipertensão arterial sistêmica. Revista Diálogos Acadêmicos, v.1, n.6, p.3-9, 2018.

PADOIN, K; COMARELLA, L.; SOLDA, C.. Medicamentos comumente prescritos na odontologia e suas principais interações medicamentosas: revisão de literatura. Journal of Oral Investigations, v.7, n.1, p.62-76, 2018.

PIRES, A. B.; MADEIRA, A. C. A.; D'ARAÚJO, K. M.; SOUZA, L. D. G.; VALADÃO, A. F.; MOTTA, P. G.. Reações adversas na cavidade oral em decorrência do uso de medicamentos. SalusVita, v.36, n.1, 2017.

PORTER, S. R.; MERCADANTE, V.; FEDELE, S.. Oral manifestations of systemic disease. Br Dent J, v.9, n.223, p. 683-691, 2017. DOI: http://doi.org/10.1038/sj.bdj.2017.884
RENNER, A. T.. Inefficiencies in a healthcare system with a regulatory split of power: a spatial panel data analysis of avoidable hospitalisations in Austria. Eur J Health Econ, v.1 n.21, p.85-104, 2020. DOI: http://doi.org/10.1007/s10198$\underline{019-01113-7}$

RIBEIRO, J.; CARNIEL, V.; SMIALOSKI, A. C.; BORTOLLI, L. A.; ROCHA, R. D. S.; MUNIZ, M. S.; ALMEIDA, W. I. S.; DIRSCHNABEL, A. J.. Doença periodontal e alterações cardiovasculares: revisão de literatura. Ação Odonto, v.1, 2016.

RUTTER-LOCHER, Z.; SMITH, T. O.; GILES, I.; SOFAT, N. Association between Systemic Lupus Erythematosus and Periodontitis: A Systematic Review and Meta-analysis. Front Immunol, v.8, p.1295, 2017. DOI: http://doi.org/10.3389/fimmu.2017.01295

SOUSA, A. L. L.; BATISTA, S. R.; SOUSA, A. C.; PACHECO, J. A. S.; VITORINO, P. V. O.; PAHOTTO, V.. Prevalência, tratamento e controle da Hipertensão Arterial em idosos de uma capital brasileira. Arq. Bras. Cardiol, v.3, n.112, p.271278, 2019. DOI: https://doi.org/10.5935/abc.20180274

TSE, S. Y.. Diabetes mellitus and periodontal disease: awareness and practice among doctors working in public general out-patient clinics in Kowloon West Cluster of Hong Kong. BMC Fam Pract, v.1 n.19, p.199, 2018. DOI: http://doi.org/10.1186/s12875-018-0887-2

VAISHNAVI, V. V. K.; SIVADAS, G.. Physician and dental surgeon's roles in diagnosing hypertension in association with Lichen Planus and Geographic Tongue - the perspective of a clinician. South African Dental Journal, v.10, n.74, p. 528-529, 2019.

VERISSÍMO, C. I. G.. Terapêutica anti-hipertensora em medicina dentária: manifestações na cavidade oral. Dissertação (Mestrado Integrado em Medicina Dentária) Instituto Superior de Ciências Da Saúde Egas Moniz, Lisboa, 2017.

WINNING, L.; LINDEN, G. J.. Periodontitis and Systemic Disease: association or Causality?. Curr Oral Health Rep, v. 1, n.4, p.1-7, 2017. DOI: http://doi.org/10.1007/s40496-017$\underline{0121-7}$

YAMA, P. A.; VALIDO, Y. R.; RAVELO, O. Q.. La diabetes mellitus y su relación con las enfermedades cardiovasculares. CorSalud, v.3, n.5, p.318-320, 2013.

A CBPC - Companhia Brasileira de Produção Científica (CNPJ: 11.221.422/0001-03) detém os direitos materiais desta publicação. Os direitos referem-se à publicação do trabalho em qualquer parte do mundo, incluindo os direitos às renovações, expansões e disseminações da contribuição, bem como outros direitos subsidiários. Todos os trabalhos publicados eletronicamente poderão posteriormente ser publicados em coletâneas impressas sob coordenação da Sustenere Publishing, da Companhia Brasileira de Produção Científica e seus parceiros autorizados. Os (as) autores (as) preservam os direitos autorais, mas não têm permissão para a publicação da contribuição em outro meio, impresso ou digital, em português ou em tradução. 